\title{
Making Holes in the Hyperspace of Subcontinua of Some Continua
}

\author{
José G. Anaya, Enrique Castañeda-Alvarado, Fernando Orozco-Zitli \\ Facultad de Ciencias, Universidad Autónoma del Estado de México, Toluca, México \\ Email: \{jgao,eca\}@uaemex.mx, forozcozitli@gmail.com
}

Received November 25, 2011; revised December 23, 2011; accepted December 30, 2011

\begin{abstract}
Let $X$ be a metric continuum. Let $A \in C(X), A$ is said to make a hole in $C(X)$, if $C(X)-\{A\}$ is not unicoherent. In this paper, we characterize elements $A \in C(X)$ such that $A$ makes a hole in $C(X)$, where $X$ is either a smooth fan or an Elsa continuum.
\end{abstract}

Keywords: Continuum; Elsa Continuum; Fan; Hyperspace; Property b); Unicoherence; Whitney Map

\section{Introduction}

A connected topological space $Z$ is unicoherent if whenever $Z=A \cup B$, where $A$ and $B$ are connected and closed subsets of $Z$, the set $A \cap B$ is connected. Let $Z$ be a unicoherent topological space and let $Z$ be an element of $Z$. We say that $Z$ makes a hole in $Z$ if $Z-\{z\}$ is not unicoherent. A compactum is a nondegenerate compact metric space. A continuum is a connected compactum with metric $d$. Given a continuum $X$, the hyperspace of all nonempty subcontinua of $X$ is denoted by $C(X)$ and it is considered with the Hausdorff metric. It is known that the hyperspace $C(X)$ is unicoherent (see [1, Theorem 19.8, p. 159]).

In the papers [2] and [3] the author present some partial solution to the following problem.

Problem. Let $\mathcal{H}(X)$ be a hyperspace of $X$ such that $\mathcal{H}(X)$ is unicoherent. For which elements,

$A \in \mathcal{H}(X)$, does $A$ make a hole in $\mathcal{H}(X)$ ?

In the current paper we present the solution to that problem when $X$ is either a smooth fan or an Elsa continuum and $\mathcal{H}(X)=C(X)$.

\section{Preliminary}

We use $\mathbb{N}$ and $\mathbb{R}$ to denote the set of positive integers and the set of real numbers, respectively. Let $Z$ be a topological space and let $A$ be a subset of $Z$. We denote $\operatorname{int}(A)$ the interior of $A$ in $Z$. An $\operatorname{arc}$ is any homeomorphic space to the closed unit interval $[0,1]$. Let $p, q$ in a topological space $Z,[p, q]$ will denote an arc, where $p$ and $q$ are the end points of $[p, q]$. A free $\operatorname{arc}$ in a continuum $X$ is an arc $[p, q]$ such that $[p, q]-\{p, q\}$ is open in $X$. A point $z$ in a connected topological space $Z$ is a cut point of (non-cut point of) $Z$ provided that $Z-\{z\}$ is disconnected (is connected). A map is a continuous function. A map $f: Z \rightarrow S^{1}$, where $Z$ is a connected topological space and $S^{1}$ is the unit circle in the Euclidean plane $\mathbb{R}^{2}$, has a lifting if there exists a map $h: Z \rightarrow \mathbb{R}$ such that $f=\exp h$, where exp is the map from $\mathbb{R}$ onto $S^{1}$ defined by $\exp (t)=(\cos (2 \pi t), \sin (2 \pi t))$. A connected topological space $Z$ has property b) if each map $f: Z \rightarrow S^{1}$ has a lifting.

By an end point of $X$, we mean an end point in the classical sense, which means a point $p$ of $X$ that is a non-cut point of any arc in $X$ that contains $p$. A subspace $Y$ of a topological space $Z$ is a deformation retract of $Z$ if there exists a map $H: Z \times I \rightarrow Z$ such that, for each $x \in Z, H(x, 0)=x, H(Z \times\{1\})=Y$ and, for each $y \in Y, H(y, 1)=y$. We say that a topological space $Z$ is contractible if there exists $z \in Z$, such that $\{z\}$ is a deformation retract of $Z$. It is known that each contractible normal topological space has property b), and so it is unicoherent (see [4, Theorems 2 and 3, pp. 69 and 70]).

\section{Smooth Fans}

A point $p$ of a continuum $X$ is a ramification point provided that $p$ is a point which is a common end point of three or more arcs in $X$ that are otherwise disjoint. A fan is an arcwise connected, hereditarily unicoherent continuum with exactly one ramification point (hereditarily unicoherent means each subcontinuum is unicoherent). The ramification point of a fan will be called the vertex of the fan. If $X$ is a fan and $x, y \in X$, then 
$[x, y]$ denotes the unique arc joining $x$ and $y$. A fan $X$ with vertex $v$ is said to be smooth provided that if $\left\{x_{n}\right\}_{n=1}^{\infty}$ is a sequence in $X$ such that it converges to a point $x \in X$, then the sequence $\left\{\left[v, x_{n}\right]\right\}_{n=1}^{\infty}$ converges to $[v, x]$ in $C(X)$.

To establish some notation, let $X$ be a smooth fan with vertex $v$ and let $E(X)=\left\{e_{i}: i \in \Delta\right\}$ be its endpoints set, where $\Delta$ is an infinity indexing set. It follows from definition of smoothness that the set:

$$
N[C(X)]=\{[v, x]: x \in X\}
$$

is a natural homeomorphic copy of $X$ in $C(X)$. By the smoothness of $X$, we have that the set:

$$
T[C(X)]=\bigcup_{i \in \Delta} C\left(\left[v, e_{i}\right]\right)
$$

is a closed subspace of $C(X)$. Furthermore, each hyperspace $C\left(\left[v, e_{i}\right]\right)$ is a 2-cell and

$$
C\left(\left[v, e_{i}\right]\right) \cap C\left(\left[v, e_{j}\right]\right)=\{v\} \text { for each } i, j \in \Delta \text { which }
$$

are different. The set of all elements of $C(X)$ such that it contains $v$ will be denoted by $C(\{v\}, X)$.

Let $A \in C(X)$. We say that $A$ is a simple arc if $A$ is an arc such that $A \cap E(X)=\varnothing$ and, there exists a sequence $\left\{A_{n}\right\}_{n=1}^{\infty}$ of $C(X)$ satisfying the following properties:

1) $A=\lim A_{n}$ and

2) for each $n \in \mathbb{N}$,

a) $v \notin A_{n}$,

b) $\operatorname{int}\left(A_{n}\right) \neq \varnothing$ and

c) $A \cap A_{n} \neq \varnothing$.

Since $X$ is embeded in the Cantor fan (see [5]), we can regard $X$ as embedded in the Euclidean plane $\mathbb{R}^{2}$ such that $v=(0,0)$ and each $\left[v, e_{i}\right]$ is a convex arc, where $e_{i} \in E(X)$. Note that for $r=0, r e_{i}=v$ for each $e_{i} \in E(X)$. Throughout this section $h$ will denote the map from $X \times[0,1]$ onto $T[C(X)]$ defined by $h(x, t)=[t x, x]$. We assume in this section that if $[a, b] \subset\left[v, e_{i}\right]$, then the distance between $v$ and $a$

is less than the distance between $v$ and $b$.

Lemma 3.1. Let $X$ be a smooth fan with vertex $v$. If $[a, b]$ is an arc contained in $\left[v, e_{i_{0}}\right]$, where $e_{i_{0}} \in E(X)$, then:

1) If $\operatorname{int}([a, b])=\varnothing$, there exists a sequence $\left\{x_{n}\right\}_{n=1}^{\infty}$ of $X$ such that $[v, b]=\lim \left[v, x_{n}\right]$ and, for each $n \in \mathbb{N}, \quad x_{n} \notin\left[v, e_{i_{0}}\right]$.

2) If $e_{i_{0}} \notin[a, b]$ and $\operatorname{int}([a, b]) \neq \varnothing$, then $\left[b, e_{i_{0}}\right]$ is a free $\operatorname{arc}$ in $X$.

Proof. The proof of (1) is easy.

In order to prove (2), we suppose that $\left[b, e_{i_{0}}\right]$ is not a free arc in $X$. Then there exists $y_{0} \in\left[b, e_{i_{0}}\right]-\left\{b, e_{i_{0}}\right\}$ such that $y_{0} \notin \operatorname{int}\left(\left[b, e_{i_{0}}\right]\right)$. Hence, $y_{0} \notin \operatorname{int}\left(\left[v, e_{i_{0}}\right]\right)$. Then, there exists a sequence $\left\{y_{n}\right\}_{n=1}^{\infty}$ of $X-\left[v, e_{i_{0}}\right]$ such that $y_{0}=\lim y_{n}$. Since $X$ a smooth fan, $\left[v, y_{0}\right]=\lim \left[v, y_{n}\right]$. Notice that $[a, b] \subset[v, b] \subset\left[v, y_{0}\right]$. Let $z_{0} \in \operatorname{int}([a, b])$. There exists a sequence $\left\{z_{n}\right\}_{n=1}^{\infty}$ of $X$ such that $z_{0}=\lim z_{n}$ and, for each $n \in \mathbb{N}$,

$z_{n} \in\left[v, y_{n}\right]$. Clearly $v \notin \operatorname{int}([a, b])$. Hence, $z_{0} \neq v$. Let $\varepsilon>0$ be such that $v \notin B_{\varepsilon}\left(z_{0}\right)$ and

$B_{\varepsilon}\left(z_{0}\right) \subset[a, b]$. Let $n_{0} \in \mathbb{N}$ be large enough such that $z_{n_{0}} \in B_{\varepsilon}\left(z_{0}\right) \subset[a, b] \subset\left[v, e_{i_{0}}\right]$. Thus,

$z_{n_{0}} \in\left[v, e_{i_{0}}\right] \cap\left[v, y_{n_{0}}\right]$. Since $X$ is a fan and $y_{n_{0}} \in X-\left[v, e_{i_{0}}\right], \quad z_{n_{0}}=v$, this is a contradiction.

Since the Hilbert cube, $Q$, is homogeneous (see [1, Theorem 11.9.1, p. 93]) and $Q-\{(1,1,1, \cdots)\}$ is contractible, we have the following result.

Lemma 3.2. Let $q \in Q$. Then $Q-\{q\}$ has property b).

Theorem 3.3. Let $X$ be a smooth fan with vertex $v$. If $A$ is a subcontinuum of $X$ such that $v \in A$ and, for each $e_{i} \in E(X), A \nsubseteq\left[v, e_{i}\right]$, then $A$ does not make a hole in $C(X)$.

Proof. We are going to prove that $C(\{v\}, X)-\{A\}$ is a deformation retract of $C(X)-\{A\}$. Notice that, for each $B \in T[C(X)]$, there exists $\left(x_{B}, t_{B}\right) \in X \times[0,1]$ such that $h\left(x_{B}, t_{B}\right)=B$. We define

$$
H=(B, t) \begin{cases}B, & \text { if } B \in C(\{v\}, X), \\ {\left[(t-1) t_{B} x_{B}, x_{B}\right],} & \text { if } B \in T[C(X)] .\end{cases}
$$

Clearly $H$ is a map. Then, $C(\{v\}, X)-\{A\}$ is a deformation retract of $C(X)-\{A\}$. Since $Q$ is homeomorphic to $C(\{v\}, X)$ (see [6, Theorem 3.1, p. 282]), $C(\{v\}, X)-\{A\}$ has property b) (see Lemma 3.2). Therefore $C(X)-\{A\}$ has property b) (see [2, Proposition 9, p. 2001]).

Lemma 3.4. Let $X$ be a smooth fan with vertex $v$ and let $[a, b] \in C\left(\left[v, e_{i_{0}}\right]\right)$ be a simple arc contained in $X$, for some $e_{i_{0}} \in E(X)$. Then

$$
\operatorname{int}\left(\left[b, e_{i_{0}}\right]\right)=\left[b, e_{i_{0}}\right]-\{b\} .
$$

Proof. Since $[a, b]$ is a simple arc, there exists a sequence $\left\{A_{n}\right\}_{n=1}^{\infty}$ of $C(X)$ that satisfies the required properties of the definition. Notice that, for each $n \in \mathbb{N}$, $A_{n} \in T[C(X)]$ and $A_{n} \subset\left[v, e_{i_{0}}\right]$. Given $n \in \mathbb{N}$, let $a_{n}, b_{n} \in\left[v, e_{i_{0}}\right]$ such that $A_{n}=\left[a_{n}, b_{n}\right]$.

We need to prove the following claim. 
Claim. $\left[b, e_{i_{0}}\right]$ is a free $\operatorname{arc}$ in $X$.

Let $n \in \mathbb{N}$. First, we suppose that there exists $n_{0} \in \mathbb{N}$ such that $b_{n_{0}} \in[v, b]$. Since $\operatorname{int}\left(\left[a_{n_{0}}, b_{n_{0}}\right]\right) \neq \varnothing$ and $e_{i_{0}} \notin\left[a_{n_{0}}, b_{n_{0}}\right],\left[b_{n_{0}}, e_{i_{0}}\right]$ is a free arc (see (2) of Lemma 3.1). Hence, $\left[b, e_{i_{0}}\right]$ is a free $\operatorname{arc}$ in $X$.

Now, we assume that, for each $n \in \mathbb{N}$, $b_{n} \in\left[b, e_{i_{0}}\right]-\{b\}$. Let $y \in\left[b, e_{i_{0}}\right]-\left\{b, e_{i_{0}}\right\}$. Notice that $b=\lim b_{n}$ and $d(b, y)>0$. Then there exists $n_{0} \in \mathbb{N}$, such that $d\left(b, b_{n_{0}}\right)<d(b, y)$. Since $\left[v, e_{i_{0}}\right]$ is a convex arc of $\mathbb{R}^{2}$, we have that $y \in\left[b_{n_{0}}, e_{i_{0}}\right]-\left\{b_{n_{0}}, e_{i_{0}}\right\}$. Since $\operatorname{int}\left(A_{n_{0}}\right) \neq \varnothing$, $\left[b_{n_{0}}, e_{i_{0}}\right]$ is a free arc in $X$ (see (2) of Lemma 3.1). Thus, $\left[b_{n_{0}}, e_{i_{0}}\right]-\left\{b_{n_{0}}, e_{i_{0}}\right\}$ is an open subset of $X$ such that $y \in\left[b_{n_{0}}, e_{i_{0}}\right]-\left\{b_{n_{0}}, e_{i_{0}}\right\} \subset\left[b, e_{i_{0}}\right]-\left\{b, e_{i_{0}}\right\}$. Hence $\left[b, e_{i_{0}}\right]-\left\{b, e_{i_{0}}\right\}$ is an open subset of $X$. This proves the claim.

By Claim, $\left[b, e_{i_{0}}\right]$ is a free $\operatorname{arc}$ in $X$. Since $\left[v, e_{i_{0}}\right]=[v, a] \cup[a, b] \cup\left[b, e_{i_{0}}\right], b \notin \operatorname{int}\left(\left[b, e_{i_{0}}\right]\right)$. Suppose that $e_{i_{0}} \notin \operatorname{int}\left(\left[b, e_{i_{0}}\right]\right)$. Then there exists a sequence $\left\{y_{m}\right\}_{m=1}^{\infty}$ in $X$ such that $e_{i_{0}}=\lim y_{m}$ and, for each $m \in \mathbb{N}, y_{m} \notin\left[b, e_{i_{0}}\right]$. Since

$\left[v, e_{i_{0}}\right]=[v, a] \cup[a, b] \cup\left[b, e_{i_{0}}\right], d\left(b, e_{i_{0}}\right)>0$ and

$e_{i_{0}}=\lim y_{m}$, we may assume that, for each $m \in \mathbb{N}$, $y_{m} \notin\left[v, e_{i_{0}}\right]$. Since $X$ is a smooth fan,

$\left[v, e_{i_{0}}\right]=\lim \left[v, y_{m}\right]$. Let $z_{0} \in\left[b, e_{i_{0}}\right]-\left\{b, e_{i_{0}}\right\} \subset\left[v, e_{i_{0}}\right]$.

There exists a sequence $\left\{z_{m}\right\}_{m=1}^{\infty}$ such that, $z_{0}=\lim z_{m}$ and, for each $m \in \mathbb{N}, z_{m} \in\left[v, y_{m}\right]$. Since $\left[b, e_{i_{0}}\right]-\left\{b, e_{i_{0}}\right\}$ is an open set in $X$ and $z_{0} \in\left[b, e_{i_{0}}\right]-\left\{b, e_{i_{0}}\right\}$, there exists $m_{0} \in \mathbb{N}$ such that $z_{m_{0}} \in\left[b, e_{i_{0}}\right]-\left\{b, e_{i_{0}}\right\} \subset\left[v, e_{i_{0}}\right]$. Then $z_{m_{0}} \in\left[v, e_{i_{0}}\right] \cap\left[v, y_{m}\right]=\{v\}$, this is a contradiction. Therefore int $\left(\left[b, e_{i_{0}}\right]\right)=\left[b, e_{i_{0}}\right]-\{b\}$.

Theorem 3.5. Let $X$ be a smooth fan with vertex $\{v\}$. If $A \in C(X)$ is a simple arc, then $A$ makes a hole in $C(X)$.
Proof. We may assume that $A=[a, b] \in C\left(\left[v, e_{i_{0}}\right]\right)$, where $e_{i_{0}} \in E(X)$ and $t_{0} \in[0,1]$ such that $a=t_{0} b$. Let:

$$
\mathcal{A}=\left(C(\{v\}, X) \cup\left(T\left[C\left(X-\operatorname{int}\left(\left[b, e_{i_{0}}\right]\right)\right)\right]\right)-\{A\}\right.
$$

and

$$
\mathcal{B}=h\left(\left[b, e_{i_{0}}\right] \times[0,1]\right)-\{A\} .
$$

By Lemma 3.4, $X-\operatorname{int}\left(\left[b, e_{i_{0}}\right]\right)$ is a smooth fan. Then $\left(T\left[C\left(X-\operatorname{int}\left(\left[b, e_{i_{0}}\right]\right)\right)\right]\right)-\{A\}$ is a connected and closed subset of $C(X)-\{A\}$. So, $\mathcal{A}$ is a connected and closed subset of $C(X)-\{A\}$.

Notice that $\mathcal{B}$ is homeomorphic to $\left[b, e_{i_{0}}\right] \times[0,1]-\left\{\left(b, t_{0}\right)\right\}$. Since $\left[b, e_{i_{0}}\right] \times[0,1]-\left\{\left(b, t_{0}\right)\right\}$ is a connected subset of $X \times[0,1]-\left\{\left(b, t_{0}\right)\right\}$, we have that $\mathcal{B}$ is a connected subset of $C(X)-\{A\}$. Clearly $\mathcal{B}$ is a closed subset of $C(X)-\{A\}$.

Notice that $C(X)-\{[a, b]\}=\mathcal{A} \cup \mathcal{B}$ and:

$$
\mathcal{A} \cap \mathcal{B}=h\left((\{b\} \times[0,1])-\left\{\left(b, t_{0}\right)\right\}\right) \cup h\left(\left[b, e_{i_{0}}\right] \times\{0\}\right) .
$$

Let:

$$
\mathcal{F}_{1}=\left(h\left(\left(\{b\} \times\left[0, t_{0}\right]\right)-\left\{\left(b, t_{0}\right)\right\}\right)\right) \cup h\left(\left[b, e_{i_{0}}\right] \times\{0\}\right)
$$

and

$$
\mathcal{F}_{2}=h\left(\left(\{b\} \times\left[t_{0}, 1\right]\right)-\left\{\left(b, t_{0}\right)\right\}\right) .
$$

Clearly, $\mathcal{F}_{1} \cup \mathcal{F}_{2}=\mathcal{A} \cap \mathcal{B}$ is a separation of $\mathcal{A} \cap \mathcal{B}$. Then $C(X)-\{A\}$ is not unicoherent.

Theorem 3.6. Let $X$ be a smooth fan with vertex $v$, let $e_{i_{0}} \in E(X)$ and let $a \in\left[v, e_{i_{0}}\right]-\left\{e_{i_{0}}\right\}$. Then $\left[a, e_{i_{0}}\right]$ does not make a hole in $C(X)$.

Proof. Let

$$
G:\left(C(X)-\left\{\left[a, e_{i_{0}}\right]\right\}\right) \times[0,1] \rightarrow C(X)-\left\{\left[a, e_{i_{0}}\right]\right\}
$$

be defined by:

$$
G(A, t)=\{t a: a \in A\} .
$$

It is easy to prove that $G$ is well defined. In order to show that $G$ is continuous, we define $G^{\prime}: C(X) \times[0,1] \rightarrow C(X)$ by $G^{\prime}(A, t)=\{t a: a \in A\}$. We prove that $G$ is continuous. Let $\left\{\left(A_{n}, t_{n}\right)\right\}_{n=1}^{\infty}$ be a sequence in $C(X) \times[0,1]$ and $\left(A_{0}, t_{0}\right) \in C(X) \times[0,1]$ such that $\left(A_{0}, t_{0}\right)=\lim \left(A_{n}, t_{n}\right)$. We suppose that there exists $B \in C(X)$ such that $B=\lim G^{\prime}\left(A_{n}, t_{n}\right)$. We will show $B=G^{\prime}\left(A_{0}, t_{0}\right)$. Let $b \in B$. Consider two sequences $\left\{b_{n}\right\}_{n=1}^{\infty}$ and $\left\{a_{n}\right\}_{n=1}^{\infty}$ of $X$ such that 
$b=\lim b_{n}$ and, for each $n \in \mathbb{N}, b_{n} \in G^{\prime}\left(A_{n}, t_{n}\right)$,

$a_{n} \in A_{n}$ and $b_{n}=t_{n} a_{n}$. Taking subsequences if necessary, we may assume that there exists $a_{0} \in X$ such that $a_{0}=\lim a_{n}$. Then $a_{0} \in A_{0}$. Moreover,

$t_{0} a_{0}=\lim t_{n} a_{n}=b$ and, so $b \in G^{\prime}\left(A_{0}, t_{0}\right)$. This proves that $B \subset G^{\prime}\left(A_{0}, t_{0}\right)$. Now, let $t_{0} a_{0} \in G^{\prime}\left(A_{0}, t_{0}\right)$. Then $a_{0} \in A_{0}$. Then there exists a sequence $\left\{a_{n}\right\}_{n=1}^{\infty}$ in $X$ such that $a_{0}=\lim a_{n}$ and, for each $n \in \mathbb{N}, a_{n} \in A_{n}$. So $t_{0} a_{0}=\lim t_{n} a_{n}$. Since, for each $n \in \mathbb{N}$,

$t_{n} a_{n} \in G^{\prime}\left(A_{n}, t_{n}\right), t_{0} a_{0} \in B$. Thus $B=G^{\prime}\left(A_{0}, t_{0}\right)$.

Hence, $G$ is a map. So $\{v\}$ is a deformation retract of $C(X)-\left\{\left[a, e_{i_{0}}\right]\right\}$.

Then $C(X)-\left\{\left[a, e_{i_{0}}\right]\right\}$ is contractible. Therefore $C(X)-\left\{\left[a, e_{i_{0}}\right]\right\}$ has property b) (see [2, Proposition 9 , p. 2001]).

Theorem 3.7. Let $X$ be a smooth fan with vertex $v$, let $e_{i_{0}} \in E(X)$ and let $[a, b] \in C\left(\left[v, e_{i_{0}}\right]\right)$ such that $e_{i_{0}} \notin[a, b]$ and $\left[b, e_{i_{0}}\right]$ is not a free arc of $X$. Then $[a, b]$ does not make a hole in $C(X)$.

Proof. In light of Proposition 9 of [2, p. 2001], it suffices to prove that there exist two connected, closed subsets $\mathcal{D}$ and $\varepsilon$ of $C(X)-\{[a, b]\}$ which have property $b)$ and the intersection of them is connected.

We may assume that there exists $t_{0} \in[0,1]$ such that $t_{0} b=a$.

We consider two cases.

Case 1. $t_{0}=0$.

Then $[a, b]=[v, b]$. Let $\mathcal{D}=T[C(X)]-\{[a, b]\}$ and $\varepsilon=C(\{v\}, X)-\{[a, b]\}$. Clearly $\mathcal{D}$ has property b).

By Theorem 3.1 of [6, p. 282], $C(\{v\}, X)$ is a Hilbert cube. By Lemma 3.2, $\varepsilon$ has property b). Notice that $(\mathcal{D} \cap \varepsilon)-\{[a, b]\}=N[C(X)]-\{[a, b]\}$. Clearly $N[C(X)]-\{[a, b]\}$ is homeomorphic to $X-\{b\}$.

Since $X-\{b\}$ is connected, $(\mathcal{D} \cap \varepsilon)-\{[a, b]\}$ is connected. By Proposition 8 of [2],

$C(X)-\{[a, b]\}=(\mathcal{D} \cup \varepsilon)-\{[a, b]\}$ has property b).

Case 2. $t_{0}>0$. Consider the following sets:

$$
\mathcal{D}=h\left(X \times\left[t_{0}, 1\right]-\left\{\left(b, t_{0}\right)\right\}\right)
$$

and

$$
\varepsilon=C(\{v\}, X) \cup h\left(X \times\left[0, t_{0}\right]-\left\{\left(b, t_{0}\right)\right\}\right) .
$$

Clearly $\mathcal{D}$ and $\varepsilon$ are connected, closed subsets of $C(X)-\{[a, b]\}$ and $\mathcal{D} \cap \varepsilon=h\left(X \times\left\{t_{0}\right\}-\left\{\left(b, t_{0}\right)\right\}\right)$.

Notice that $\mathcal{D} \cap \varepsilon$ is homeomorphic to $X-\{b\}$. So, since $X-\{b\}$ is connected, $\mathcal{D} \cap \varepsilon$ is connected.

Now, we are going to prove that $\mathcal{D}$ and $\varepsilon$ have property b). If we define $H: \mathcal{D} \times[0,1] \rightarrow \mathcal{D}$ by $H(h(x, t), s)=h(x, t+(1-t) s)$, we have $h(X \times\{1\})$ is a deformation retract of $\mathcal{D}$. Since $h(X \times\{1\})$ is contractible, $h(X \times\{1\})$ has property b) (see [2, Proposition 9 , p. 2001]). Hence, $\mathcal{D}$ has property b) (see [2, Proposition 9, p. 2001]).

In order to prove that $\varepsilon$ has property b), note that $C(\{v\}, X)$ is a deformation retract of $\varepsilon$. By Theorem 3.1 of [6, p. 282], $C(\{v\}, X)$ is homeomorphic to a Hilbert cube. Thus, $C(\{v\}, X)$ has property b). Hence, $\varepsilon$ has property b) (see Proposition 9 of [2, p. 2001]). Therefore $C(X)-\{[a, b]\}=\mathcal{D} \cup \mathcal{\varepsilon}$ has property b).

\section{Classification}

Theorem 3.8. Let $X$ be a smooth fan with vertex $v$ and $A \in C(X)$. Then $A$ makes a hole in $C(X)$ if and only if $A$ a simple arc.

Proof. Let $A \in C(X)$ be such that $A$ makes a hole in $C(X)$. By Theorem 3 of [2, p. 2001] and by Theorem 3.3, $A$ is an arc $[p, q]$. By Theorems 3.6 and 3.7, $[p, q] \subset\left[v, e_{i_{0}}\right]-\left\{v, e_{i_{0}}\right\}$ for some $e_{i_{0}} \in E(X)$, and $\left[q, e_{i_{0}}\right]$ is a free arc in $X$. In order to prove that $[p, q]$ is a simple arc, let $\left\{a_{n}\right\}_{n=1}^{\infty},\left\{b_{n}\right\}_{n=1}^{\infty}$ be sequences in $[p, q]-\{p, q\}$ and $\left[q, e_{i_{0}}\right]-\{q\}$, respectively, such that $p=\lim a_{n}$ and $q=\lim b_{n}$. Then

$[p, q]=\lim \left[a_{n}, b_{n}\right]$ and, for each $n \in \mathbb{N}, v \notin\left[a_{n}, b_{n}\right]$, $\operatorname{int}\left(\left[a_{n}, b_{n}\right]\right) \neq \varnothing$ and $[p, q] \cap\left[a_{n}, b_{n}\right] \neq \varnothing$. Therefore $A$ is a simple arc.

The sufficiency follows from Theorem 3.5.

\section{Elsa Continua}

A compactification of $[0, \infty)$ with an arc as the remainder is called an Elsa continuum. The Elsa continua was defined by S. B. Nadler Jr., in [7]. A particular example of an Elsa continuum is the familiar $\sin (1 / \mathrm{x})$ continuum. There are uncountably many topologically different Elsa continua, the different topological types being a consequence of different ways $[0, \infty)$ "patterns into" the remainder of the compactification [8, p. 184]. Let $X$ be a continuum. A Whitney map for $C(X)$ is a continuous function $\mu: C(X) \rightarrow[0,1]$ that satisfies the following two conditions:

1) for any $A, B \in C(X)$ such that $A \subset B$ and $A \neq B, \mu(A)<\mu(B)$,

2) $\mu(\{x\})=0$ for each $x \in X$ and c) $\mu(X)=1$.

A Whitney block in $C(X)$, respectively a Whitney level in $C(X)$, is a set of the form $\mu^{-1}([s, t])$, respectively $\mu^{-1}(t)$, where $0 \leq s \leq t \leq 1$. It is known that Whitney maps always exist (see [1, Theorem 13.4, p. 107]). Moreover, Whitney blocks and Whitney levels in 
$C(X)$ are continua (see [1, Theorem 19.9, p. 160]).

Throughout this section $X=I \cup R$ will denote a Elsa continuum, where $I$ is the remainder of $X$ and $R$ is homeomorphic to the half-ray $[0, \infty)$.

Lemma 4.1. Let $\mu: C(X) \rightarrow \mathbb{R}$ be a Whitney map, let $t \in(0, \mu(I)]$ and let $A_{0} \in \mu^{-1}(t) \cap C(I)$. Then $\mu^{-1}([0, t])-\left\{A_{0}\right\}$ has property $\left.b\right)$.

Proof. We consider $\mu_{1}=\left.\mu\right|_{C(I)}$. It is easy to prove that $\mathcal{A}=\mu_{1}^{-1}([0, t])-\left\{A_{0}\right\}$ has property b).

Let $\mathcal{B}=\mathcal{A} \cup \mu^{-1}(0)$. Since $\mu^{-1}(0)$ has property b) (see $\left[9,12.66\right.$, p. 269]) and $\mathcal{A} \cap \mu^{-1}(0)=\mu_{1}^{-1}(0), \mathcal{B}$ has property b) (see [2, Proposition 8, p. 2001]).

Let $f: \mu^{-1}([0, t])-\left\{A_{0}\right\} \rightarrow S^{1}$ be a map. Then there exists a map $h_{0}: \mathcal{B} \rightarrow \mathbb{R}$ such that expo $h_{0}=\left.f\right|_{\mathcal{B}}$.

Given $A \in \mu^{-1}((0, t]) \cap C(R)$, it is an arc contained in $R$ and it is determined by its end point, $i_{A}$, lying near to the end point of $R$. Let $\alpha_{A}$ be an order arc in $C(X)$ from $i_{A}$ to $A$. Since $\alpha$ has property $b$ ), there exists a map $h_{A}: \alpha \rightarrow \mathbb{R}$ such that $\exp \circ h_{A}=\left.f\right|_{\alpha}$ and $h_{A}\left(\left\{i_{A}\right\}\right)=h_{0}\left(\left\{i_{A}\right\}\right)$.

We define $h: \mu^{-1}([0, t])-\left\{A_{0}\right\} \rightarrow \mathbb{R}$ by

$$
h(A) \begin{cases}h_{0}(A), & \text { if } A \in \mathcal{B}, \\ h_{A}(A), & \text { if } A \in \mu^{-1} T([0, t]) \cap C(R) .\end{cases}
$$

In order to prove that $h$ is continuous, let $\{B\}_{n=1}^{\infty}$ be a sequence of $\mu^{-1}([0, t])-\left\{A_{0}\right\}$ such that $\lim B_{n}=B_{0}$ for some $B_{0} \in \mu^{-1}([0, t])-\left\{A_{0}\right\}$. We consider two cases.

Case 1. For each $n \in \mathbb{N}, B_{n} \in \mathcal{B}$.

Since $\mathcal{B}$ is a closed subset of $\mu^{-1}([0, t])-\left\{A_{0}\right\}$, $B_{n} \in \mathcal{B}$. Then $\operatorname{limh}\left(B_{n}\right)=h\left(B_{0}\right)$.

Case 2. For each $n \in \mathbb{N}, B_{n}$ is an arc contained in $R$.

We consider two subcases.

Subcase 1. $B_{0} \subset R$.

Let $p \in R$ be such that $\bigcup_{n=0}^{\infty} B_{n} \subset[p, q]$, where $q$ denotes the end point of $R$. Then $\left.\mu\right|_{C([p, q])}$ is a Whitney map for $C([p, q])$. Since $\left(\left.\mu\right|_{C([p, q])}\right)^{-1}(0)$ is an arc, it has property b). By Lemma 4 of [10, p. 254],

$\left(\left.\mu\right|_{C([p, q])}\right)^{-1}([0, t])$ has property b). Then there exists a map $g:\left(\left.\mu\right|_{C([p, q])}\right)^{-1}([0, t]) \rightarrow \mathbb{R}$ such that

expog=f $\left.\right|_{C([p, q])}$ and $g\left(\left\{i_{B_{1}}\right\}\right)=h_{0}\left(\left\{i_{B_{1}}\right\}\right)$. Notice that $\left.h_{0}\right|_{(\mu \mid C([p, q]))^{-1}(0)}$ and $\left.g\right|_{(\mu \mid C([p, q]))}{ }^{-1}(0)$ are liftings of $\left.f\right|_{(\mu \mid C([p, q]))^{-1}(0)}$ and $g\left(\left\{i_{B_{1}}\right\}\right)=h_{0}\left(\left\{i_{B_{1}}\right\}\right)$. Then $\left.h_{0}\right|_{(\mu \mid C([p, q]))^{-1}(0)}=\left.g\right|_{(\mu \mid C([p, q]))^{-1}(0)}$.

Given $n \in \mathbb{N} \cup\{0\}$. Notice that $\left.h_{B_{n}} \quad y \quad g\right|_{\alpha_{B n}}$ are liftliftings of $\left.f\right|_{\alpha_{B n}}$ and $h_{B_{n}}\left(i_{B_{n}}\right)=h_{0}\left(i_{B_{n}}\right)=\left.g\right|_{\alpha_{B n}}\left(i_{B_{n}}\right)$. Then $h_{B_{n}}=\left.g\right|_{\alpha_{B_{n}}}$. Hence,

$$
\begin{aligned}
h\left(B_{0}\right) & =h_{B_{0}}\left(B_{0}\right)=g\left(B_{0}\right)=\lim g\left(B_{n}\right) \\
& =\lim h_{B_{n}}\left(B_{n}\right)=\lim h\left(B_{n}\right) .
\end{aligned}
$$

Subcase 2. $B_{0} \subset I$.

We can consider that, for any $n, m \in \mathbb{N}, i_{B_{n}} \neq i_{B_{m}}$, if $n \neq m$.

Since $B_{0}=\lim B_{n}$ and $X$ is a compact space, we may assume that there exists $i_{B_{0}} \in B_{0}$ such that $i_{B_{0}}=\lim i_{B_{n}}$. We can suppose, taking subsequence if it is necessary, that there exists a subcontinuum $\alpha_{B_{0}}$ of $C(X)$ such that $\alpha_{B_{0}}=\lim \alpha_{B_{n}}$. It is easy to show that $\alpha_{B_{0}}$ is either an order arc from $b_{0}$ to $B_{0}$ or a one point-set.

Given $n \in \mathbb{N} \cup\{0\}$, we have $\left.\mu\right|_{\alpha_{B n}}$ is an homeomorphism between $\left[0, \mu\left(B_{n}\right)\right]$ and $\alpha_{B_{n}}$. Let

$g_{n}:\left[0, \mu\left(B_{n}\right)\right] \rightarrow \alpha_{B_{n}}$ be such that $g_{n}=\left(\left.\mu\right|_{\alpha_{B n}}\right)^{-1}$.

By Lemma 3.1 of [7, p. 330], we can assume that $X$ is a subset of $\mathbb{R}^{2}$. Let

$$
D=X \times\{0\} \cup\left(\bigcup_{n=0}^{\infty}\left(\left\{i_{B_{n}}\right\} \times\left[0, \mu\left(B_{n}\right)\right]\right)\right) .
$$

Notice that $D$ is a subset of the Euclidian space $\mathbb{R}^{3}$ and $X \times\{0\}$ is a deformation retract of $D$. Then $D$ has property $b)$.

We define $f_{1}: D \rightarrow \mu^{-1}\left(\left[0, t_{0}\right]\right)$ by

$$
f_{1}(x, t)= \begin{cases}x & \text { if } t=0, \\ g_{n}(t), & \text { if }(x, t) \in\left\{i_{B_{n}}\right\} \times\left[0, \mu\left(B_{n}\right)\right] .\end{cases}
$$

It is easy to prove that $f_{1}$ is a map. Since $D$ has property b), there exists a map $h_{3}: D \rightarrow \mathbb{R}$ such that expoh$h_{3}=f \circ f$ and $h_{3}\left(i_{B_{1}}, 0\right)=h_{0}\left(f_{1}\left(i_{B_{1}}, 0\right)\right)_{1}$. Then $\left.h_{3}\right|_{X \times\{0\}}=\left.h_{0} \circ f_{1}\right|_{X \times\{0\}}$. Thus, given $n \in \mathbb{N} \cup\{0\}$, it can prove that $\left.h_{3}\right|_{\left\{B_{\left.B_{n}\right\}}\right\}\left[0, \mu\left(B_{n}\right)\right]}=\left.h_{B_{n}} \circ f_{1}\right|_{\left\{i_{\left.B_{n}\right\}}\right\} \times\left[0, \mu\left(B_{n}\right)\right]}$. Hence, $h\left(B_{0}\right)=\lim h\left(B_{n}\right)$.

This proves that $\mu^{-1}([0, t])-\left\{A_{0}\right\}$ has property $\left.b\right)$.

Theorem 4.2. Let $X=I \cup R$ be an Elsa continuum and let $A \in C(I)$. Then $A$ does not make a hole in $C(X)$.

Proof. In light of Proposition 8 of [2], it suffices to prove that there exist two connected and closed subsets $\mathcal{A}$ and $\mathcal{B}$ of $C(X)-\{A\}$, which have property b) and the intersection of them is connected.

Let $\mu: C(X) \rightarrow[0,1]$ be a Whitney map. Let 
$t=\mu(A), \mathcal{A}=\mu^{-1}([t, 1])-\{A\}$ and

$\mathcal{B}=\mu^{-1}([0, t])-\{A\}$. Clearly $\mathcal{A} \cup \mathcal{B}=C(X)-\{A\}$,

$\mathcal{A}$ and $\mathcal{B}$ are connected and closed subsets of

$C(X)-\{A\}$.

By Lemma 13 of [2, p. 2004], $\mathcal{A}$ has property b) and, by Lemma $4.1, \mathcal{B}$ has property b).

In order to show that $\mathcal{A} \cap \mathcal{B}$ is connected, notice that $\mathcal{A} \cap \mathcal{B}=\mu^{-1}(\{t\})-\{A\}$ and $A \in \mu^{-1}(\{t\}) \cap C(I)$. By Corollary 3 of $[11$, p. 386],

$\mu^{-1}(\{t\})=\left(\mu^{-1}(\{t\}) \cap C(I)\right) \cup\left(\mu^{-1}(\{t\}) \cap C(R)\right)$ and $\mu^{-1}(\{t\}) \cap C(R)$ approximates the whole continuum $\mu^{-1}(\{t\}) \cap C(I)$. Hence, $\mathcal{A} \cap \mathcal{B}$ is connected.

Theorem 4.3. Let $X=I \cup R$ be an Elsa continua. If $A \in C(X)$ such that $A$ is homeomorphic to $X$, then $A$ does not make a hole in $C(X)$.

Proof. In light of Proposition 2.4 of [3, p. 3], it suffices to prove that there exists a closed neighborhood $\mathcal{W}$ of $A$ in $C(X)$ such that $\mathcal{W}-\{A\}$ has property b) and $b d_{C(X)}(\mathcal{W})$ is connected $\left(b d_{C(X)}(\mathcal{W})\right.$ denotes the boundary of $\mathcal{W}$ in $C(X))$.

Let $\mu: C(X) \rightarrow[0,1]$ be a Whitney map. Let $\mathcal{W}=\mu^{-1}([\mu(I), 1])$. Clearly $\mathcal{W}$ is a closed neighborhood of $A$. Since $b d_{C(X)}(\mathcal{W})=\mu^{-1}(\mu(I))$, $b d_{C(X)}(\mathcal{W})$ is connected. By [12, Theorem 4.3, p. 217], $\mathcal{W}$ is a 2-cell. Moreover, $A$ is an element of its manifold boundary (see [11, Lemma 2, p. 386]). Then $\mathcal{W}-\{A\}$ is contractible. Therefore $\mathcal{W}-\{A\}$ has property b) (see [2, Proposition 9, p. 2001]).

\section{Classification}

Theorem 4.4. Let $X=I \cup R$ be an Elsa continuum and let $A \in C(X)$. Then $A$ makes a hole in $C(X)$ if and only if $A$ is a free arc $p q$ such that $p, q \notin \operatorname{int}(p q)$.

Proof. Let $A \in C(X)$ be such that $A$ makes a hole in $C(X)$. By Theorem 3 of [2, p. 2001] and Theorems 4.2 and 4.3, $A$ is an arc $p q$ contained in $R$. So, $A$ is a free arc in $X$. By Theorem 4 of [2, p. 2001], $p, q \notin \operatorname{int}(p q)$.
The sufficiency follows from Theorem 1 of $[2, p$. 2001].

\section{REFERENCES}

[1] A. Illanes and S. B. Nadler Jr., "Hyperspaces: Fundamentals and Recent Advances," Marcel Dekker, Inc., New York, 1999.

[2] J. G. Anaya, "Making Holes in Hyperspaces," Topology and Its Applications, Vol. 154, No. 10, 2007, pp. 20002008. doi:10.1016/j.topol.2006.09.017

[3] J. G. Anaya, "Making Holes in the Hyperspace of Subcontinua of a Peano Continuum," Topology Proceedings, Vol. 37, 2011, pp. 1-14.

[4] S. Eilenberg, "Transformations Continues en Circonférence et la Topologie du Plan," Fundamenta Mathematicae, Vol. 26, 1936, pp. 61-112.

[5] C. Eberhart, "A Note on Smooth Fans," Colloquium Mathematicum, Vol. 20, 1969, pp. 89-90.

[6] C. Eberhart and S. B. Nadler Jr., "Hyperspaces of Cones and Fans," Proceedings of the American Mathematical Society, Vol. 77, No. 22, 1979, pp. 279-288. doi:10.1090/S0002-9939-1979-0542098-5

[7] S. B. Nadler Jr., "Continua Whose Cone and Hyperspace Are Homeomorphic," Transactions of the American Mathematical Society, Vol. 230, 1977, pp. 321-345. doi:10.1090/S0002-9947-1977-0464191-0

[8] S. B. Nadler Jr., "Arc Components of Certain Chainable Continua," Canadian Mathematical Bulletin, Vol. 14, No. 2, 1971, pp. 183-189. doi:10.4153/CMB-1971-033-8

[9] S. B. Nadler Jr., "Continuum Theory: An Introduction," Marcel Dekker, Inc., New York, 1992.

[10] A. Illanes, "Multicoherence of Whitney Levels," Topology and Its Applications, Vol. 68, No. 3, 1996, pp. 251-265. doi:10.1016/0166-8641(95)00064-X

[11] W. J. Charatonik, "Some Counterexamples Concerning Whitney Levels," Bulletin of the Polish Academy of Sciences Mathematics, Vol. 31, 1983, pp. 385-391.

[12] C. B. Hughes, "Some properties of Whitney continua in the hyperspace C(X)," Topology Proceedings, Vol. 1, 1976, pp. 209-219. 